\title{
Long-term Performance of Epic and PERIMOUNT Mitral Bioprostheses in Young Rheumatics
}

Lakshmi Kumari Sankhyan ${ }^{1}$, Ujjwal Kumar Chowdhury ${ }^{2 *}$, Niwin George ${ }^{2}$, Suruchi Hasija ${ }^{2}$, Srikant Sharma ${ }^{2}$, Niraj Nirmal Pandey ${ }^{3}$, Sai Divya Yadavalli ${ }^{2}$, Maroof A. Khan',

${ }^{1}$ Department of Cardiothoracic and Vascular Surgery, All India Institute of Medical Sciences, Bilaspur, Himachal Pradesh

${ }^{2}$ Departments of Cardiothoracic and Vascular Surgery,

${ }^{3}$ Cardiac Radiology

${ }^{4}$ Biostatistics, All India Institute of Medical Sciences, New Delhi

*Corresponding Author: Ujjwal Kumar Chowdhury, MCh, Diplomate NB Professor Department of CTVS AIIMS, India.

Received Date: February 11, 2021; Accepted Date: March 22, 2021; Published Date: March 29,2021

Citation: Lakshmi K. Sankhyan., Ujjwal K. Chowdhury., Niwin George, Suruchi Hasija, Srikant Sharma, et al. (2021) Long-term Performance of Epic and PERIMOUNT Mitral Bioprostheses in Young Rheumatics. J. Clinical Cardiology and Cardiovascular Interventions, 4(6);

Doi:10.31579/2641-0419/142

Copyright: (c) 2021 Ujjwal Kumar Chowdhury, This is an open-access article distributed under the terms of the Creative Commons Attribution License, which permits unrestricted use, distribution, and reproduction in any medium, provided the original author and source are credited.

\begin{abstract}
Objective: We compared the long-term cossmposites of valve-related reoperation, morbidity and mortality following two types of mitral bioprostheses in young rheumatics aged $\leq 45$ years.

Methods: Retrospective comparative analysis of structural valve-related reoperations, and survival data were performed on 260 propensity matched patients, undergoing bioprosthetic MVR between 2000 and 2019, using Epic (Group I, n=130) or PERIMOUNT bioprostheses (Group II, n=130).

Results: The median age was 34.5 (IQR: 28-39) and 34 (IQR: 29-40) years for group I and II respectively. Hazard regression for mortality included HR (95\% CI) preoperative congestive heart failure (CHF) 4.70 (1.7612.56), $\mathrm{p}=0.002$; renal failure 66.91 (12.88-347.59), $\mathrm{p}<0.0001$; low left ventricular ejection fraction $\leq 0.25,3.76$ (1.72-7.27), $\mathrm{p}=0.004$; and valve-related reoperations 3.82 (1.81-9.56), $\mathrm{p}=0.001$. Although the structural valve degeneration (SVD)-related reoperations were more among the PERIMOUNT group, propensity score matching did not exhibit any difference between the groups [8.5\% (Group I) vs 14.6\% (Group II), SMD -0.23, p=0.5]. At a median follow-up of 134 (IQR: 99.5-178.5) months, actuarial survival was $96.36 \% \pm 0.01 \%(93.11-98.10)$, and there was no difference in survival between the groups $(\log$ rank $\mathrm{p}=0.70)$.
\end{abstract}

Conclusions: Both Epic and PERIMOUNT mitral bioprostheses provide similar long-term clinical outcomes and are an appealing alternative to mechanical prosthesis in younger rheumatics.

Key words: mitral valve replacement; mitral bioprostheses; structural valve deterioration; propensity score matching

Running title: Mitral bioprostheses in young rheumatics

\section{Introduction}

Valve replacement in young patients entails a choice between a mechanical prosthesis with a $1 \%$ to $3 \%$ per year life-long bleeding risk versus a bioprosthesis with limited long-term durability necessitating eventual reoperation. [1-5]

In developing countries, severity and rapid progression of rheumatic heart disease (RHD) in young adults precludes repair in the great majority and the criterions of selection of one prosthesis over other remains debatable. [1-5]

Over the last 20 years, there is a shift away from a clear cut age limit towards patients' wish and lifestyle considerations. [6-10] This may be related to the enhanced durability of new-generation bioprostheses, improved outcomes of redo surgery, or development of valve-in-valve transcatheter valve implantation. [6-10]

Currently, 18- to 20-year valve durability experience with mitral bioprostheses is available, [6-11] but little is known regarding composites of complications, namely valve-related reoperations, morbidity, and mortality in young rheumatics. [12]

We earlier evaluated the mid-term outcome of PERIMOUNT mitral bioprosthesis in young rheumatics with respect to survival, thromboembolism, structural valve deterioration (SVD), and quality of life. [10] In the current study, we analysed the long-term results of 
bioprosthetic mitral valve replacement (MVR) in young rheumatics aged $<45$ years using Epic and PERIMOUNT mitral bioprostheses.

The primary objective was to assess the long-term outcomes of composites of valve-related complications between Epic or PERIMOUNT mitral bioprostheses in young rheumatics aged $<45$ years. The outcome variables assessed were valve-related reoperations, morbidity, and mortality. The secondary objectives were to: i) compare the short- and long-term outcomes including SVD, and ii) ascertain duration and intensity of anticoagulation treatment required in immediate and late postoperative period and before re-replacement of degenerated bioprostheses.

Methods
This retrospective study conforms to the principles outlined in the declaration of Helsinki. Institutional review board approval was available and informed consent was obtained from all patients.

\section{Patient selection criteria}

Selection of a mechanical or bioprosthetic MVR was determined by patients' preference and surgeon's judgement based on patients' age, predisposition to bleeding, life-style, and compliance to anticoagulant therapy. Young rheumatics aged $<45$ years undergoing isolated MVR with or without tricuspid annuoplasty using either Epic or PERIMOUNT bioprostheses were included in this descriptive case series (Figures 1A, 1B).

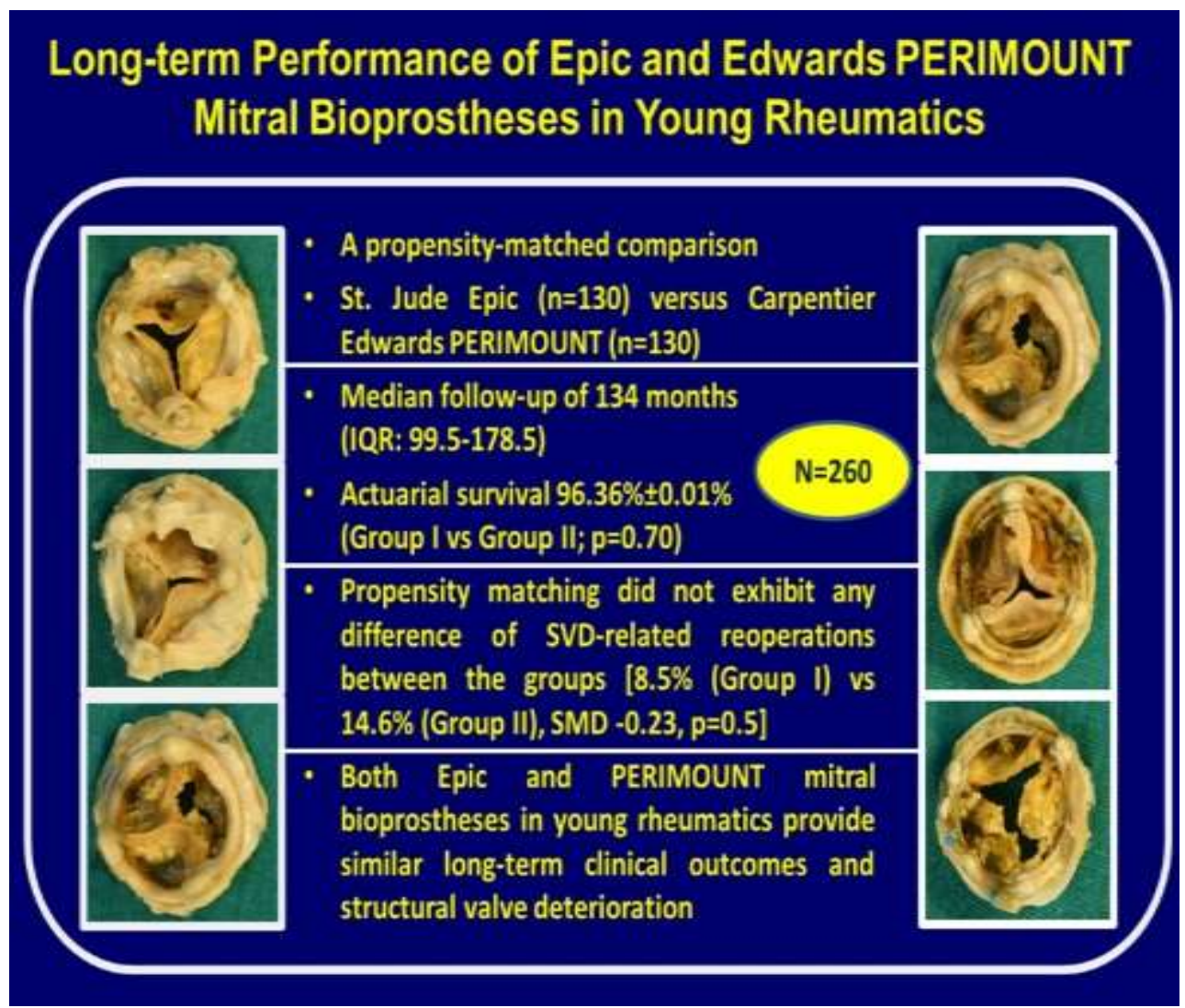




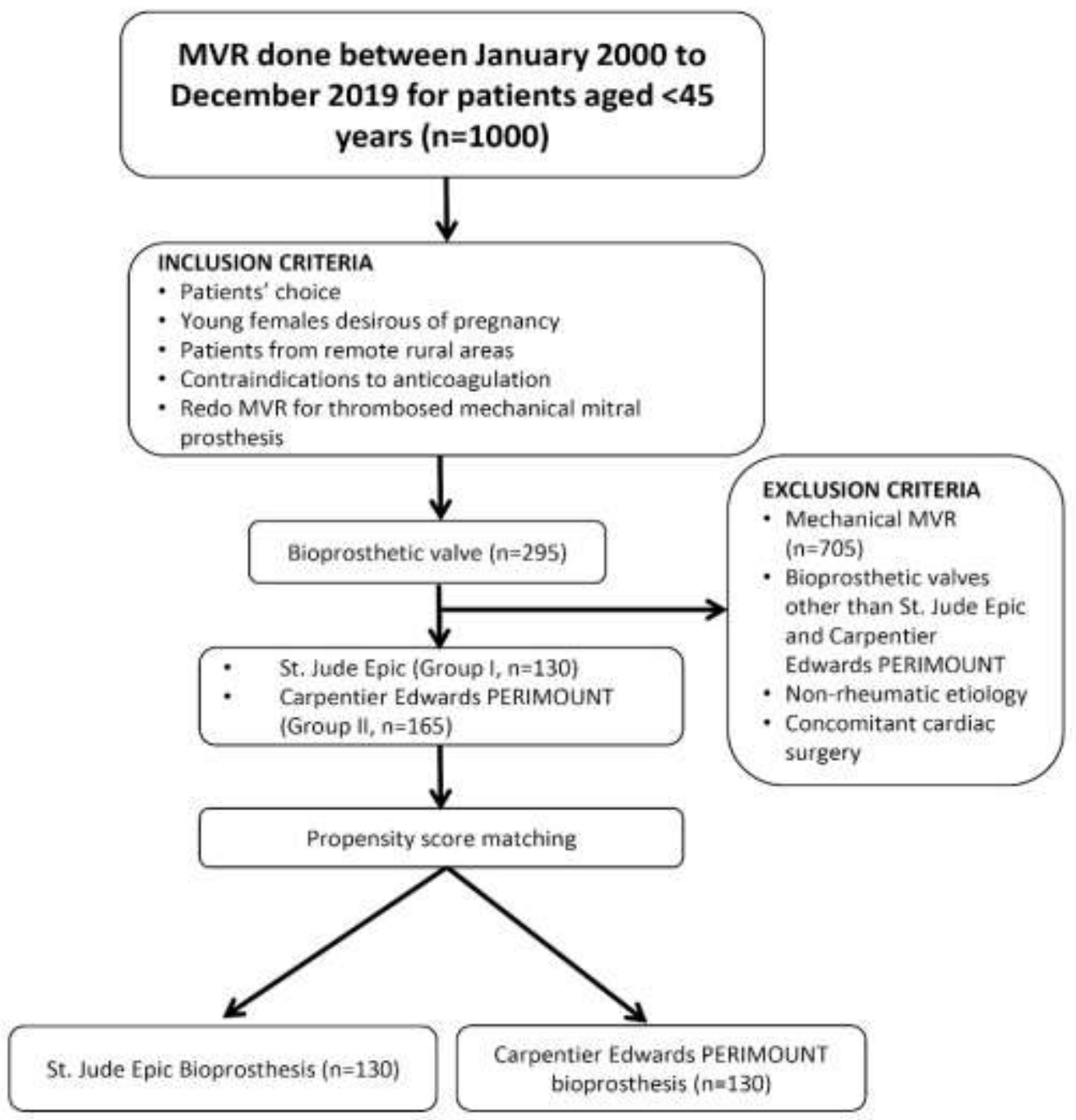

Figure 1B: Consort diagram showing inclusion and exclusion criteria for young rheumatics aged $<45$ years undergoing MVR using either Epic (Group I) or PERIMOUNT (group II) bioprostheses.

Preoperatively, $88(67.7 \%)$ patients of group I and $91(70 \%)$ patients of group II had atrial fibrillation.

Patients undergoing MVR using prosthesis other than mentioned above, non-rheumatic etiology, and concomitant cardiac surgeries were excluded. Young females desirous of pregnancy, patients coming from remote rural areas making follow-up and anticoagulant monitoring practically difficult, contraindications to use of anticoagulation, patients with thrombosed mechanical mitral prosthesis, and patients' choice were indications for bioprosthetic MVR.

Our institutional policy is to use bioprostheses beyond 18-years of age only after bone growth and maturation are completed. In this study, one patient aged 12-years with a thrombosed mechanical prosthesis and another patient aged 13-years with thalassemia and hemolysis underwent bioprosthetic MVR.
In patients with mitral stenosis and a small left ventricle, PERIMOUNT prosthesis with long struts although implanted was not usually preferred and the low-profile Epic bioprosthesis was chosen and was part of the propensity score. Both surgeon's preference and a small left ventricle were determinants of using an Epic bioprosthesis. This was a retrospective review of medical records of young rheumatics aged $<45$ years undergoing bioprosthetic MVR by the corresponding author at a single centre over 19 years (January 2000 to December 2019).

A cohort of 295 consecutive patients (Group I, $n=130$; Group II, $n=165$ ) was propensity score matched and resulted in $n=130$ (Group I, St. Jude Epic) and n=130 (Group II, Carpentier-Edwards PERIMOUNT model 6900 mitral- Edwards Lifesciences, Baxter Healthcare Corporation, Irvine, CA, USA) propensity matched patients. Among these, 132 patients of PERIMOUNT were from our previous investigation and 33 new patients were added (Figures 2A, 2B). 

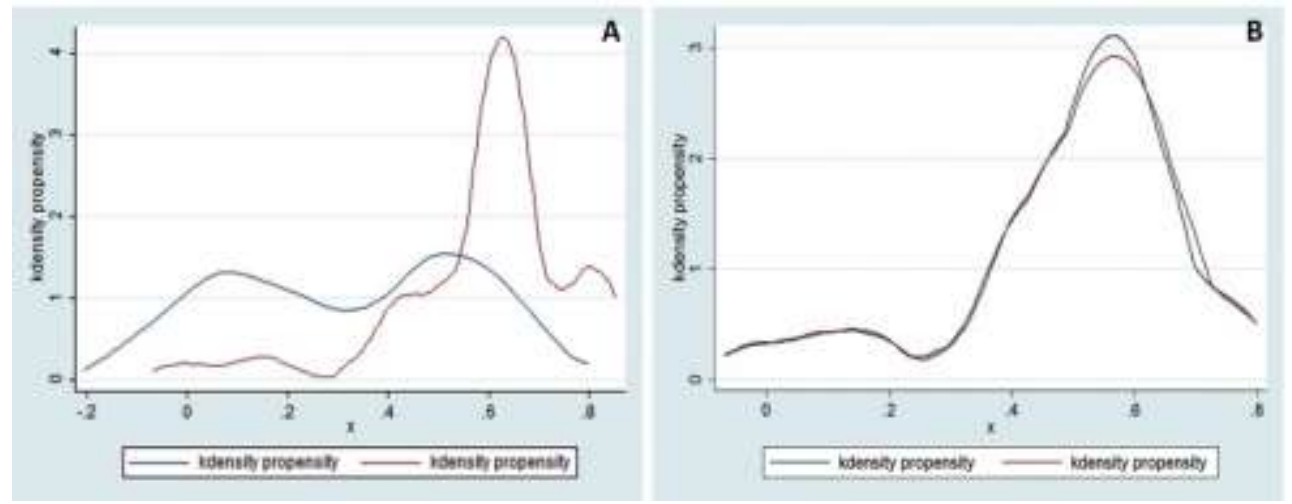

Figures 2A, 2B: Propensity density graph of patients undergoing Epic and PERIMOUNT bioprosthetic MVR before (2A) and after (2B) propensity score matching.

\section{Surgical techniques}

The technical details of surgical steps have been enumerated in the video presentation (Video Presentation) as well as in our earlier publications.[3,2,10,13] Every attempt was made to preserve the chordopapillary apparatus ensuring implantation of an appropriate sized prosthesis without leaflet entrapment or LVOTO. Total chordo-papillary apparatus was preserved using Milki's technique whenever feasible (Group I, $n=40,30.8 \%$; Group II, $n=46,35.4 \%$ ). In patients with calcified leaflets with annular extension and severe subvalvular fusion, the mitral apparatus was completely excised (Group I, n=80, 61.5\%; Group II, $\mathrm{n}=74,56.9 \%$ ). The remaining patients had only posterior chordal preservation (Group I, n=10, 7.7\%; Group II, n=10, 7.7\%).

The technical details of chordal preservation, annulus decalcification and its effect on regional and global ventricular function have been addressed in our previous publications.[3,5,10,13] Size of the bioprosthetic valve ranged from $25 \mathrm{~mm}$ to $33 \mathrm{~mm}$ [valve size: $33 \mathrm{~mm}$ (Group I, n=7; Group II, n=7); 31 mm (Group I, n=14; Group II, n=16); 29 mm (Group I, n=35; Group II, n=34); 27 mm (Group I, n=57; Group II, n=56); 25 mm (Group I, $n=17$; Group II, $n=17)$ ]. Intraoperative transoesophageal echocardiography was performed to confirm satisfactory prosthetic valve function immediately after surgery.

Patients undergoing redo MVR for degenerated bioprostheses $(n=30)$ were subjected to a uniform surgical protocol standardised by the corresponding author. A mechanical heart valve [(Medtronic Open PivotTM AP $360^{\circ}$ Apex and AP, Medtronic Inc., Mx, USA); size 24mm: 12 patients, $26 \mathrm{~mm}$ : 10 patients; St. Jude Medical Inc. St. Paul, Minn, 27mm: 4 patients, $29 \mathrm{~mm}$ : 4 patients] was used in patients undergoing explantation of degenerated bioprosthesis (Video presentation), (Figures 3A, 3B).

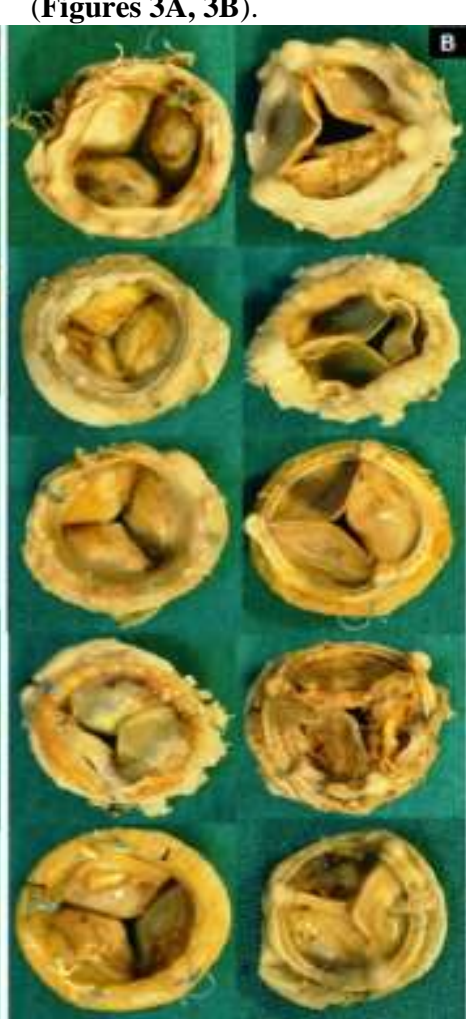

Figure 3A, 3B: Photographs of explanted Epic (Figure 3A) and PERIMOUNT (Figure 3B) mitral bioprostheses showing different types of structural valve deterioration (Cuspal perforation, tear, thickening, calcification, stiffness, wear and abrasions, creep, and stress fracture) [A-Atrial surface; BVentricular surface) 
Six-monthly follow-up data included clinical history, NYHA class assessment, and any valve-related events. [14,15] If 6-monthly evaluation was not possible after repeated attempts to contact the patient, it was considered missing. If two consecutive evaluations were missing, the patient was considered lost to follow-up. Transthoracic twodimensional (2D), colour flow and Doppler echocardiography was performed according to American Society of Echocardiography criteria within first six months and then annually. [15]

\section{Definitions (Electronics)}

\section{Outcome measures}

Valve-related mortality included death caused by thrombosis, thromboembolism, hemorrhage, SVD, non-structural dysfunction, or prosthetic valve endocarditis and death related to reoperation for a valverelated complication including sudden unexplained, unexpected deaths. Valve-related mortality was defined either as early/perioperative (i.e. in hospital or within 30 days of operation) or late (after 30 days) attributed to the explanted valve. [15]

Valve-related morbidity was defined in this study as permanent valverelated impairment as a result of permanent neurologic or other function deficit caused by valve thrombosis, thromboembolism, hemorrhage, SVD, non-structural dysfunction, prosthetic valve endocarditis, or reoperation. [15]

Late reoperations were defined as reoperations that occurred more than 30 days after implant. Reoperations were defined as any subsequent MVR. Reoperations that did not involve mitral valve replacement were excluded. [7]

Structural valve deterioration was diagnosed as clinically relevant valvular stenosis or insufficiency by Doppler echocardiography, reoperation, or necropsy. Examples included cuspal perforation, tear, thickening, calcification, stiffness, stretching, wear and abrasions, thinning, leaflet escape, stent creep, or stress fracture. Structural deterioration that resulted from endocarditis, paravalvular leak, or thrombosis was not included in the SVD category. [7, 15]

Stroke was defined as any cerebrovascular accident documented during the index hospitalization as well as any subsequent hospital admission in which the principal diagnosis was hemorrhagic or ischemic stroke (not including transient ischemic attacks). [7,15]
A major bleeding event was defined as any subsequent hospital admission in which the principal diagnosis was intracerebral hemorrhage, hemopericardium/cardiac tamponade, gastrointestinal hemorrhage, hematuria, hemarthrosis, hemoptysis, or retinal hemorrhage. Bleeding events were classified as major (i.e. requiring hospital admission or transfusion, of intracranial location, or causing death), intracranial, or minor (i.e. prospectively recorded but not major). $[7,15]$

Heart failure was defined as per previous publications as the composite end-point of (i) New York Heart Association (NYHA) functional class 3 or 4 for more than 4 consecutive weeks, corroborated with physical examination, chest X-Ray, ECG and echocardiography findings when available, or (ii) death where the primary or main contributing diagnosis was heart failure. [7,15]

\section{Anticoagulation}

Patients were started on warfarin and aspirin (100 mg/day) on first postoperative day maintaining an INR between 2.0 and 2.5. After discharge, patients were reviewed at one week, one month, three months, then subsequently at six months interval. INR was tested until 12 weeks after the operation and anticoagulation was stopped in patients with normal sinus rhythm.

Patients with a preoperative LA/LAA clot, history of recent thromboembolism, aneurysmal LA, atrial fibrillation, and degenerated bioprosthesis were maintained on low intensity anticoagulation with an INR between 1.5 and 1.8. Despite having aneurysmal left atrium and atrial fibrillation in the preoperative period, therapeutic anticoagulation was not required because of bioprosthetic implant. All patients received aspirin life-long, unless contraindicated. The study end-points were the composites of valve-related complications, namely mortality, morbidity, reoperations, and explantation due to SVD.

\section{Selection of a balanced cohort}

Table 1 shows the significant imbalances in baseline characteristics between patients treated with PERIMOUNT and Epic MVR before matching. To assemble a balance cohort of patients with Epic and PERIMOUNT MVR, we used propensity-score matching method on measured baseline characteristics. For this purpose, we estimated propensity scores for treatment (group) for each of the 260 patients using multivariable logistic regression model (Figure 4).

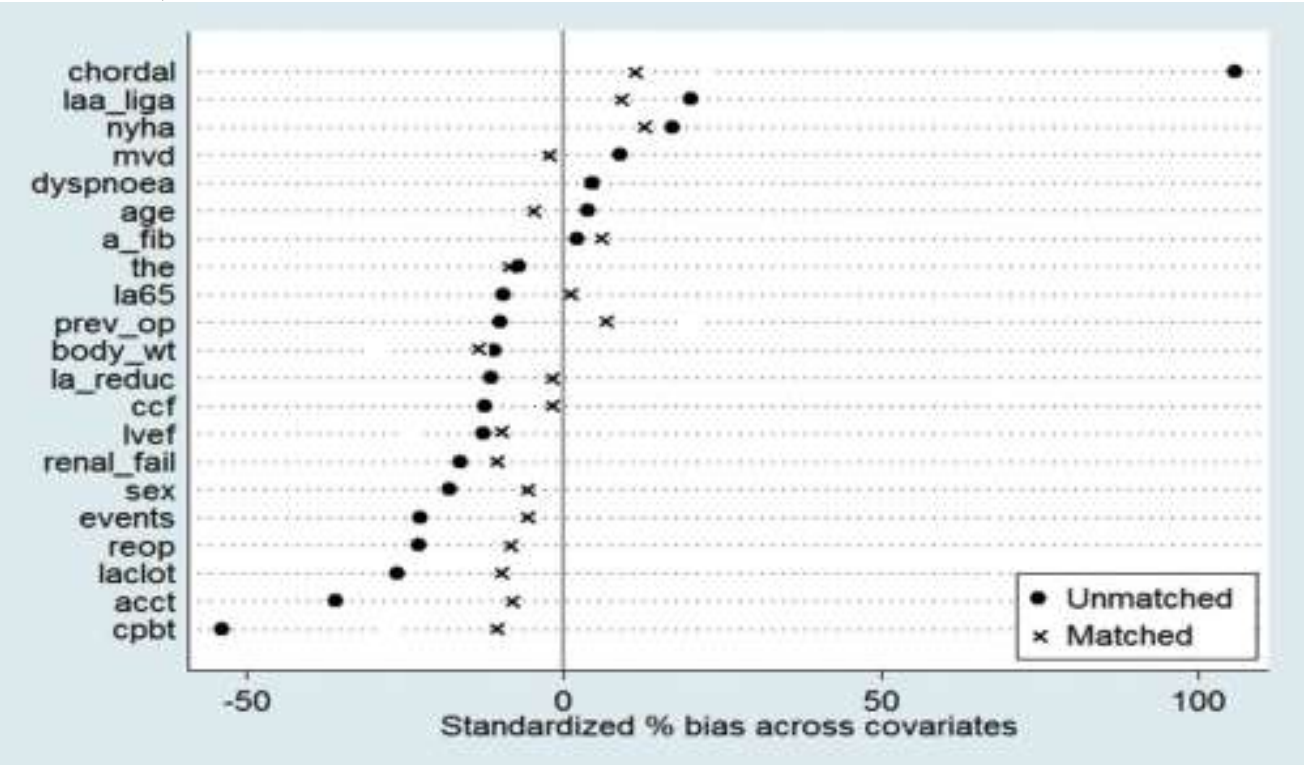


Group was used as the dependent variable and baseline characteristics namely- LA reduction, aortic cross-clamp time, thromboembolism, dyspnoea, previous operation, LVEF, chordal preservation, type of mitral valve disease were included as covariates to find the best optimal match set. Here, model's effectiveness are not important because propensity-score based models are sample-specific adjusters and are not intended to be used for out-of-sample prediction, discrimination or estimation of coefficients. The efficacy of propensity-score models is best assessed by estimating post-match absolute standardized differences between baseline covariates that directly quantifies the bias in the means or proportions of covariates across the groups. Therefore, we presented before and after propensity match standardized differences and its findings in Love plots (Figure 4). An absolute standardized difference of $0 \%$ indicates no residual bias and, $10 \%$ are considered of inconsequential bias. Greedy nearest neighbouring matching method was used for matching protocol with a caliper of 0.1 to match 1: 1 patients with Epic and PERIMOUNT mitral bioprostheses. We were able to match 130 of 165 PERIMOUNT bioprostheses patients with 130 patients of Epic

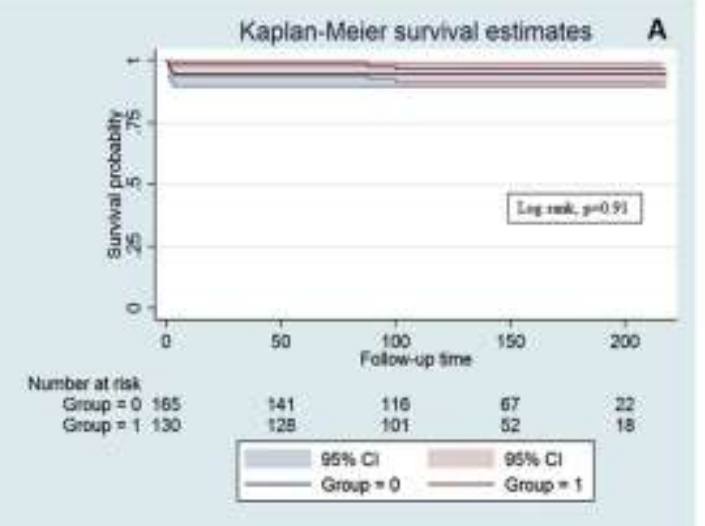

bioprostheses (Figures 2A, 2B).

\section{Statistical Analysis}

For descriptive analyses, we used Pearson Chi-square/Fisher's exact test and t-test/Wilcoxon rank-sum tests for before match and McNemar's test and paired sample t-test/sign-rank test for after match comparisons of baseline covariates between patients with Epic and PERIMOUNT MVR. Kaplan-Meier curve with 95\% confidence interval and matched Cox regression analyses were used to determine the associations of group with various outcomes during months of follow-up. All statistical analyses were done using STATA 14.0 Software (College Station, Texas, USA) and two-sided tests with a p-value of $<0.05$ were considered statistically significant.

The freedom from composites of valve-related complications, namely valve-related mortality, reoperation, and adverse postoperative events were calculated by Kaplan-Meier actuarial methods and compared with log-rank statistic (Figures 5A, 5B, 6, 7).

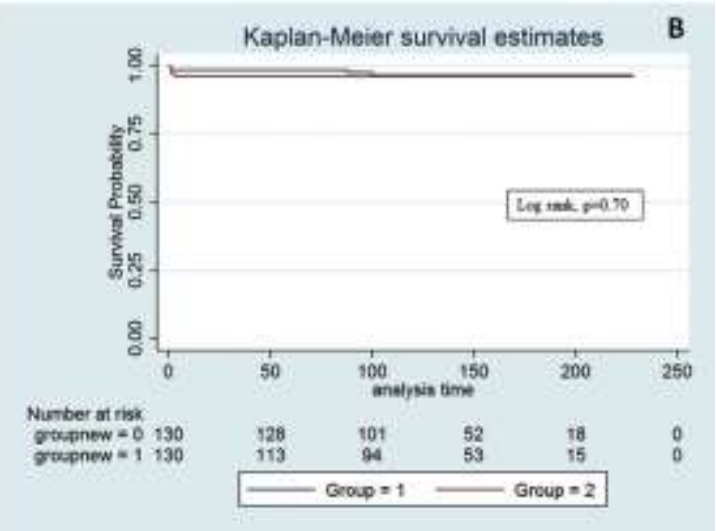

Figures 5A, 5B: Survival probability from Kaplan-Meier curve of patients before (5A) and after (5B) propensity score matching (Log rank: group I vs group II; unmatched $p=0.91$; matched $p=0.70)$.

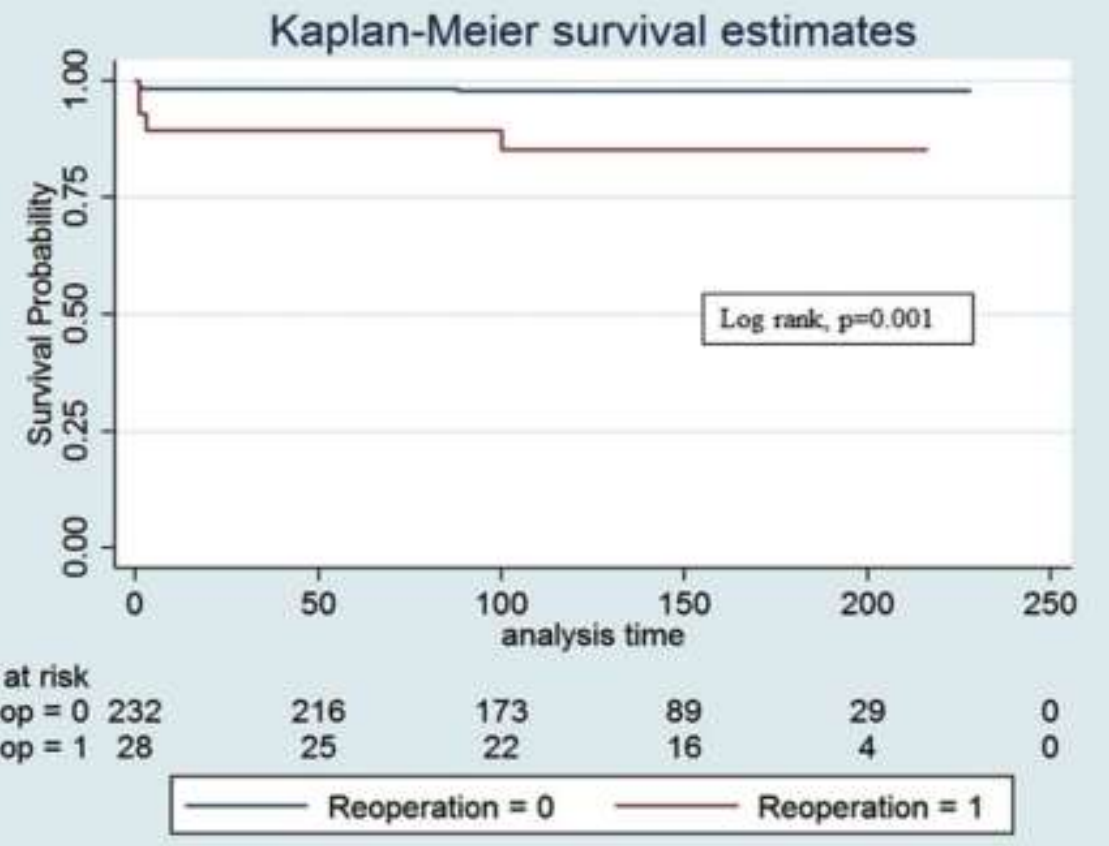

Figures 6: Survival probability from Kaplan-Meier curve of patients (Group I, $n=130$; Group II, $n=130$ ) with and without reoperations for structural valve deterioration $(n=30)$ undergoing mitral bioprosthetic implantation using Epic and PERIMOUNT bioprostheses respectively 


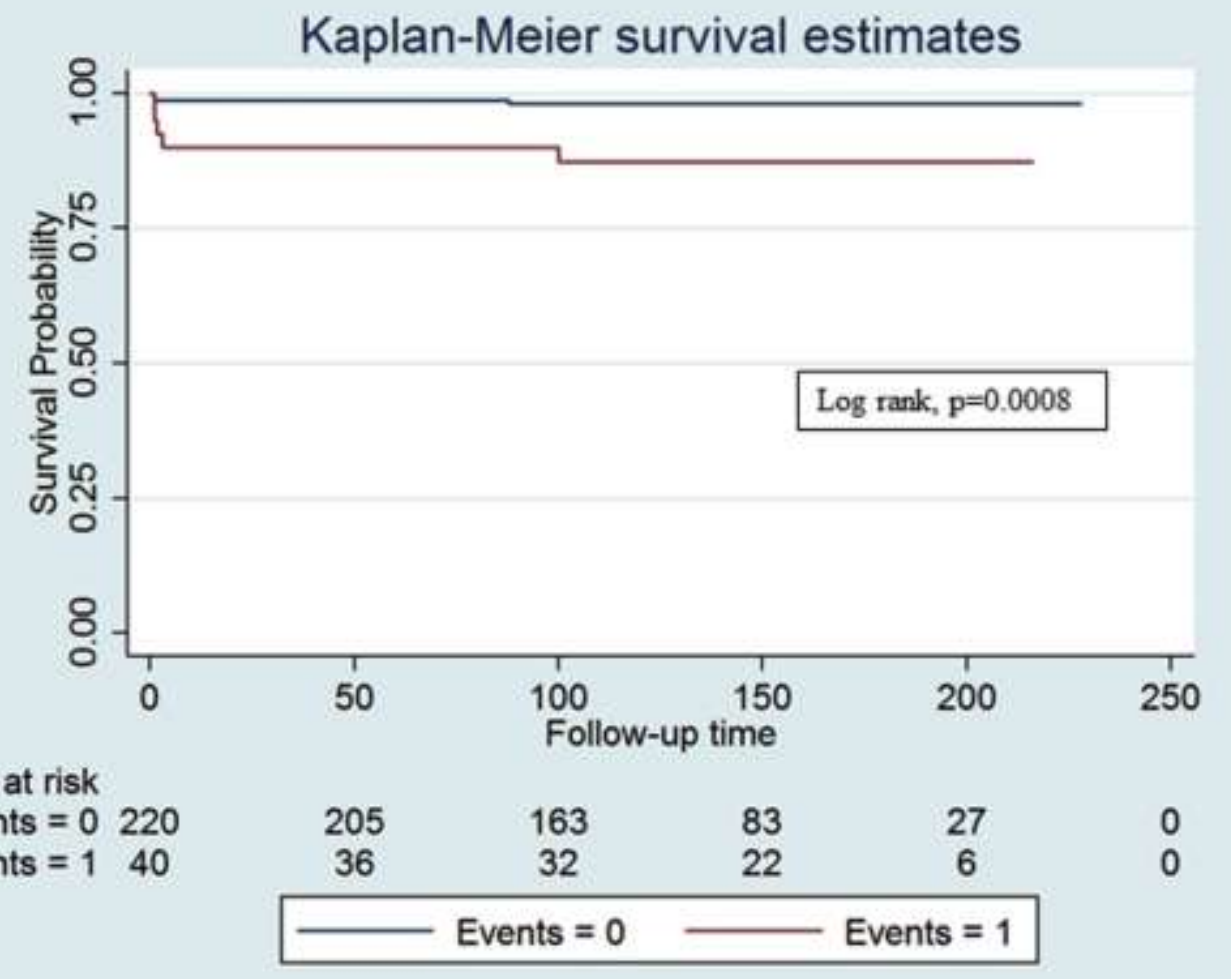

Figure 7: Survival probability from Kaplan-Meier curve of patients (Group I, $n=130$; Group II, $n=130$ ) with and without cumulative postoperative adverse events $(n=40)$ undergoing mitral bioprosthetic implantation using Epic and PERIMOUNT bioprostheses respectively

\section{Results}

\section{Study population}

After propensity matching as described previously, final study population consisted of 260 patients aged between 16 and 45 (Group I: median 34.5 (IQR: 28-39); Group II: median 34.0 (IQR: 29-40) years with no differences among the 130 matched pairs in preoperative characteristics (Table 1). The PERIMOUNT group required more reoperations for SVD [14.6\% ( $n=19)$ vs $8.5 \% \quad(n=11)$, SMD -0.23, $\mathrm{p}=0.5]$.

Median ischemic time was 30.5 minutes (IQR: 29-38) and 40.0 minutes (IQR: 32-56), ( $<<0.001)$ for Group I and II respectively. Median CPB time for Group I was 44.0 minutes (IQR: 41-66); and for Group II was 56.5 minutes (IQR: 48-72), $\mathrm{p}<0.0001$. The LAA was ligated in 235 $(90.4 \%)$ patients. Ninety-five $(36.5 \%)$ patients underwent LA reduction for giant LA. No surgery was performed for atrial fibrillation (Table 1).

\begin{tabular}{|c|c|c|c|c|c|c|c|c|}
\hline \multirow[b]{2}{*}{ Covariates } & \multicolumn{4}{|c|}{ Before Propensity Score Matching } & \multicolumn{4}{|c|}{ After Propensity Score Matching } \\
\hline & $\begin{array}{l}\text { Epic bioprosthesis } \\
\text { (Group I, } n=130 \text { ) } \\
\text { No. of patients (\%) }\end{array}$ & $\begin{array}{l}\text { PERIMOUNT } \\
\text { bioprosthesis } \\
\text { (Group II, } n=165 \text { ) } \\
\text { No. of patients (\%) }\end{array}$ & $S M D$ & $\begin{array}{l}p- \\
\text { value }\end{array}$ & $\begin{array}{l}\text { Epic } \\
\text { bioprosthesis } \\
\text { (Group I, n=130) } \\
\text { No. of patients } \\
(\%)\end{array}$ & $\begin{array}{l}\text { PERIMOUNT } \\
\text { bioprosthesis } \\
\text { (Group II, } \\
\text { n=130) } \\
\text { No. of patients } \\
(\%)\end{array}$ & $\begin{array}{l}S M \\
D\end{array}$ & $\begin{array}{l}p \text { - } \\
\text { value }\end{array}$ \\
\hline $\begin{array}{l}\text { Sex } \\
\text { Male } \\
\text { Female }\end{array}$ & $\begin{array}{l}40(30.7 \%) \\
90(69.3 \%)\end{array}$ & $\begin{array}{c}65(39.4 \%) \\
100(60.06 \%)\end{array}$ & -0.15 & 0.07 & $\begin{array}{l}40(30.7 \%) \\
90(69.3 \%)\end{array}$ & $\begin{array}{l}54(41.5 \%) \\
76(58.5 \%)\end{array}$ & $\begin{array}{c}- \\
0.18\end{array}$ & 1.38 \\
\hline $\begin{array}{l}\text { Dyspnoea } \\
\text { Yes } \\
\text { No }\end{array}$ & $\begin{array}{c}129(99.2 \%) \\
1(0.8 \%)\end{array}$ & $\begin{array}{c}163(98.7 \%) \\
2(1.3 \%)\end{array}$ & $<0.001$ & 0.68 & $\begin{array}{c}129(99.2 \%) \\
1(0.8 \%)\end{array}$ & $\begin{array}{c}129(99.2 \%) \\
1(0.8 \%)\end{array}$ & 0.04 & $<0.001$ \\
\hline $\begin{array}{l}\text { New York Heart } \\
\text { Association } \\
\text { Class IV } \\
\text { Class III }\end{array}$ & $\begin{array}{l}39(30 \%) \\
91(70 \%)\end{array}$ & $\begin{array}{c}37(22.4 \%) \\
128(77.6 \%)\end{array}$ & 0.07 & 0.09 & $\begin{array}{l}39(30 \%) \\
91(70 \%)\end{array}$ & $\begin{array}{l}37(28.5 \%) \\
93(71.5 \%)\end{array}$ & 0.17 & $<0.001$ \\
\hline $\begin{array}{l}\text { CCF on inotropes \& } \\
\text { ventilator } \\
\text { Present } \\
\text { Absent }\end{array}$ & $\begin{array}{c}17(13.1 \%) \\
113(86.9 \%)\end{array}$ & $\begin{array}{c}29(17.6 \%) \\
136(82.4 \%)\end{array}$ & 0.1 & 0.18 & $\begin{array}{c}17(13.1 \%) \\
113(86.9 \%)\end{array}$ & $\begin{array}{c}24(18.5 \%) \\
106(81.5 \%)\end{array}$ & $\overline{-}$ & 0.33 \\
\hline
\end{tabular}




\begin{tabular}{|c|c|c|c|c|c|c|c|c|}
\hline \multirow[b]{2}{*}{ Covariates } & \multicolumn{4}{|c|}{ Before Propensity Score Matching } & \multicolumn{4}{|c|}{ After Propensity Score Matching } \\
\hline & $\begin{array}{l}\text { Epic bioprosthesis } \\
\text { (Group I, } n=130) \\
\text { No. of patients }(\%)\end{array}$ & $\begin{array}{l}\text { PERIMOUNT } \\
\text { bioprosthesis } \\
\text { (Group II, } n=165) \\
\text { No. of patients (\%) }\end{array}$ & $S M D$ & $\begin{array}{l}p- \\
\text { value }\end{array}$ & $\begin{array}{l}\text { Epic } \\
\text { bioprosthesis } \\
\text { (Group I, } n=130 \text { ) } \\
\text { No. of patients } \\
(\%)\end{array}$ & $\begin{array}{l}\text { PERIMOUNT } \\
\text { bioprosthesis } \\
\text { (Group II, } \\
\text { n=130) } \\
\text { No. of patients } \\
(\%)\end{array}$ & $\begin{array}{l}S M \\
D\end{array}$ & $\begin{array}{l}p- \\
\text { value }\end{array}$ \\
\hline $\begin{array}{l}\text { Renal failure } \\
\text { requiring peritoneal/ } \\
\text { hemodialysis } \\
\text { Yes } \\
\text { No }\end{array}$ & $\begin{array}{c}3(2.3 \%) \\
127(97.7 \%)\end{array}$ & $\begin{array}{c}9(5.5 \%) \\
156(94.5 \%)\end{array}$ & 0.04 & 0.14 & $\begin{array}{c}3(2.3 \%) \\
127(97.7 \%)\end{array}$ & $\begin{array}{c}9(6.9 \%) \\
121(70.8 \%)\end{array}$ & $\begin{array}{c}- \\
0.16\end{array}$ & $<0.001$ \\
\hline $\begin{array}{l}\text { Mitral valve } \\
\text { pathology } \\
\text { MS } \\
\text { MS+MR }\end{array}$ & $\begin{array}{l}92(70.8 \%) \\
38(29.2 \%)\end{array}$ & $\begin{array}{c}55(33.3 \%) \\
110(66.7 \%)\end{array}$ & 0.16 & 0.26 & $\begin{array}{l}92(70.8 \%) \\
38(29.2 \%)\end{array}$ & $\begin{array}{l}38(29.2 \%) \\
92(70.8 \%)\end{array}$ & 0.08 & 0.76 \\
\hline $\begin{array}{l}\text { Atrial fibrillation } \\
\text { Present } \\
\text { Absent }\end{array}$ & $\begin{array}{l}88(67.7 \%) \\
42(32.3 \%)\end{array}$ & $\begin{array}{c}110(66.7 \%) \\
55(33.3 \%)\end{array}$ & 0.19 & 0.47 & $\begin{array}{l}88(67.7 \%) \\
42(32.3 \%)\end{array}$ & $\begin{array}{l}91(70 \%) \\
39(30 \%)\end{array}$ & 0.02 & 2.0 \\
\hline $\begin{array}{l}\text { Thromboembolism } \\
\text { Yes } \\
\text { No }\end{array}$ & $\begin{array}{c}10(7.7 \%) \\
120(92.3 \%)\end{array}$ & $\begin{array}{c}16(9.7 \%) \\
149(90.3 \%)\end{array}$ & 0.05 & 0.34 & $\begin{array}{c}10(7.7 \%) \\
120(92.3 \%)\end{array}$ & $\begin{array}{c}11(8.5 \%) \\
119(91.5 \%)\end{array}$ & $\overline{-}$ & 0.09 \\
\hline $\begin{array}{l}\text { Left atrial size }>65 \\
\text { Yes } \\
\text { No }\end{array}$ & $\begin{array}{l}46(35.4 \%) \\
84(64.6 \%)\end{array}$ & $\begin{array}{l}66(40 \%) \\
99(60 \%)\end{array}$ & 0.24 & 0.15 & $\begin{array}{l}46(35.4 \%) \\
84(64.6 \%)\end{array}$ & $\begin{array}{l}52(40 \%) \\
78(60 \%)\end{array}$ & $0 . \overline{39}$ & 2.3 \\
\hline $\begin{array}{l}\text { LA reduction } \\
\text { Yes } \\
\text { No }\end{array}$ & $\begin{array}{l}44(33.8 \%) \\
86(66.2 \%)\end{array}$ & $\begin{array}{c}65(39.4 \%) \\
100(60.6 \%)\end{array}$ & 0.19 & 0.17 & $\begin{array}{l}44(33.8 \%) \\
86(66.2 \%)\end{array}$ & $\begin{array}{l}51(39.2 \%) \\
79(60.8 \%)\end{array}$ & $\begin{array}{c}- \\
0.11\end{array}$ & 1.4 \\
\hline $\begin{array}{l}\text { Previous operation } \\
\text { Yes } \\
\text { No }\end{array}$ & $\begin{array}{l}31(23.8 \%) \\
99(76.2 \%)\end{array}$ & $\begin{array}{c}54(32.7 \%) \\
111(67.3 \%)\end{array}$ & 0.19 & 0.06 & $\begin{array}{l}31(23.8 \%) \\
99(76.2 \%)\end{array}$ & $\begin{array}{l}42(32.3 \%) \\
88(67.7 \%)\end{array}$ & $\overline{-}$ & 0.42 \\
\hline $\begin{array}{l}\text { Chordal } \\
\text { Yes } \\
\text { No }\end{array}$ & $\begin{array}{c}120(92.3 \%) \\
10(7.7 \%)\end{array}$ & $\begin{array}{l}82(49.7 \%) \\
83(50.3 \%)\end{array}$ & $<0.001$ & -0.000 & $\begin{array}{c}120(92.3 \%) \\
10(7.7 \%)\end{array}$ & $\begin{array}{l}60(46.1 \%) \\
70(53.9 \%)\end{array}$ & 1.06 & 0.75 \\
\hline $\begin{array}{l}\text { Reoperation } \\
\text { Yes } \\
\text { No }\end{array}$ & $\begin{array}{c}11(8.5 \%) \\
119(91.5 \%)\end{array}$ & $\begin{array}{c}23(13.9 \%) \\
142(86.1 \%)\end{array}$ & 0.04 & 0.11 & $\begin{array}{c}11(8.5 \%) \\
119(91.5 \%)\end{array}$ & $\begin{array}{c}19(14.6 \%) \\
111(85.4 \%)\end{array}$ & $\overline{-}-$ & 0.5 \\
\hline $\begin{array}{l}\text { Cumulative events } \\
\text { (postoperative) } \\
\text { Yes } \\
\text { No }\end{array}$ & $\begin{array}{c}21(16.1 \%) \\
109(83.3 \%)\end{array}$ & $\begin{array}{l}26(15.7 \%) \\
13984.3 \%)\end{array}$ & 0.05 & 0.16 & $\begin{array}{c}21(16.1 \%) \\
109(83.3 \%)\end{array}$ & $\begin{array}{c}26(20 \%) \\
141(80 \%)\end{array}$ & $-\overline{18}$ & 0.1 \\
\hline $\begin{array}{l}\text { Age (years) } \\
\quad(\text { Mean } \pm \text { SD })\end{array}$ & $33.72 \pm 6.95$ & $33.44 \pm 8.27$ & -0.75 & 0.04 & $33.7 \pm 6.95$ & $33.69 \pm 6.99$ & 0.03 & 0.78 \\
\hline $\begin{array}{r}\text { Body weight }(\mathrm{kg}) \\
(\text { Mean } \pm \text { SD) }\end{array}$ & $48.66 \pm 10.35$ & $49.9 \pm 11.9$ & 0.34 & -0.19 & $48.66 \pm 10.35$ & $50.17 \pm 11.16$ & $\begin{array}{c}- \\
0.11 \\
\end{array}$ & 0.07 \\
\hline $\begin{array}{l}\text { Preoperative left } \\
\text { ventricular ejection } \\
\text { fraction }(\%) \\
(\text { Mean } \pm \text { SD) }\end{array}$ & $47.86 \pm 19.85$ & $50.38 \pm 20.26$ & 0.28 & -0.10 & $47.86 \pm 19.85$ & $49.68 \pm 19.98$ & $-\overline{12}$ & 0.19 \\
\hline $\begin{array}{l}\text { Aortic cross clamp } \\
\text { time (minutes) } \\
\text { (Mean } \pm \text { SD) }\end{array}$ & $38.4 \pm 15.45$ & $43.62 \pm 13.4$ & 0.002 & 0.16 & $38.40 \pm 15.45$ & $43.86 \pm 13.56$ & -.36 & 0.34 \\
\hline $\begin{array}{l}\text { Cardiopulmonary } \\
\text { bypass time } \\
\text { (minutes) } \\
\quad(\text { Mean } \pm \text { SD) }\end{array}$ & $51.8 \pm 15.6$ & $59.78 \pm 13.95$ & 0.75 & -0.011 & $51.8 \pm 15.61$ & $59.65 \pm 15.0$ & 0.54 & 0.95 \\
\hline
\end{tabular}


Before Propensity Score Matching

\begin{tabular}{|l} 
Bef \\
\hline Epic bioprosthesis \\
(Group I, $\mathrm{n}=130)$ \\
No. of patients $(\%)$
\end{tabular}

Follow-up (months)

$139.71 \pm 46.5$

PERIMOUNT bioprosthesis

(Group II, n=165)

No. of patients $(\%)$

$129.5 \pm 58.6$

\begin{tabular}{|l|l|} 
SMD & $\begin{array}{l}\boldsymbol{p} \text { - } \\
\text { value }\end{array}$ \\
\hline 0.1 & 0.1 \\
\hline
\end{tabular}

\begin{tabular}{|l|l} 
& \multicolumn{1}{|c}{ After } \\
Epic \\
bioprosthesis \\
(Group I, $\mathbf{n = 1 3 0 )}$ \\
No. of patients \\
$(\%)$
\end{tabular}

After Propensity Score Matching

\begin{tabular}{|l|l|l|}
$\begin{array}{l}\text { PERIMOUNT } \\
\text { bioprosthesis } \\
\text { (Group II, } \\
\text { n=130) } \\
\text { No. of patients } \\
(\%)\end{array}$ & $\begin{array}{l}S M \\
D\end{array}$ & $\begin{array}{l}p \text { - } \\
\text { value }\end{array}$ \\
\hline $129.4 \pm 57.35$ & 0.1 & 0.1 \\
\hline
\end{tabular}

Table 1: Preoperative and intraoperative characteristics of patients undergoing bioprosthetic mitral valve replacement before and after propensity score matching in this study

\section{Operative mortality and morbidity}

There were 4 (3.1\%) hospital deaths among group I, and $5(3.8 \%)$ among group II due to LCOS after reoperations for thrombosed mechanical prostheses $(n=4)$, degenerated bioprosthesis $(n=1)$, intractable ventricular arrhythmias $(n=2)$, and sepsis $(n=2)$ in patients with left ventricular and renal failure on preoperative ventilation and renal failure. There was no differences in incidence of perioperative mortality and morbidities between the two groups (Table 2A).

\begin{tabular}{|l|c|c|c|}
\hline Outcomes & $\begin{array}{c}\text { Epic bioprosthesis (Group I, } \\
\mathbf{n = 1 3 0 )} \\
\text { No. of patients (\%) }\end{array}$ & $\begin{array}{c}\text { PERIMOUNT bioprosthesis } \\
\text { (Group II, n=130) } \\
\text { No. of patients (\%) }\end{array}$ \\
\hline Hospital mortality & $4(3.1 \%)$ & $5(3.8 \%)$ & \multicolumn{1}{c|}{ p value } \\
\hline Reoperation for bleeding & $3(2.3 \%)$ & $4(3.1 \%)$ & 0.70 \\
\hline Tracheostomy ventilator support & $3(2.3 \%)$ & $2(1.5 \%)$ & 1.0 \\
\hline Pericardiocentesis & $3(2.3 \%)$ & $5(3.8 \%)$ & 1.0 \\
\hline Transient ischemic attack & $1(0.8 \%)$ & $1(0.8 \%)$ & 0.72 \\
\hline
\end{tabular}

Table 2A: Early complications (within 30 days)

\section{Late outcomes}

Late mortality was $1.6 \%(n=2)$ in group I and $1.6 \%(n=2)$ in group II $(\mathrm{p}=1.0)$. The causes of late deaths were persistent congestive heart failure $(n=2)$, intractable ventricular arrhythmias following redo surgeries for thrombosed mechanical prosthesis $(n=1)$ and renal failure $(n=1)$ between 45 and 90 days following surgery. There was no difference in risks of hospital and late deaths between the groups.
At late follow-up, more patients were in atrial fibrillation in group II (64\% vs $54.8 \%, p=0.16)$. Only $1(0.7 \%)$ patient each of both groups developed systemic thromboembolism on $40^{\text {th }}$ and $45^{\text {th }}$ postoperative days, and was not related to low-intensity anticoagulation. There were no differences in late thromboembolic events between the two groups. Hemorrhagic complications necessitating hospitalization occurred in $1.6 \%$ of group I and $1.6 \%$ of group II $(\mathrm{p}=1.0)$, (Table $2 \mathrm{~B})$. No patients of either group presented with bioprosthesis endocarditis.

\begin{tabular}{|l|c|c|c|}
\hline Outcomes & $\begin{array}{c}\text { Epic bioprosthesis } \\
\text { (Group I, n=126) } \\
\text { No. of patients (\%) }\end{array}$ & $\begin{array}{c}\text { PERIMOUNT } \\
\text { bioprosthesis } \\
\text { (Group II, n=125) } \\
\text { No. of patients (\%) }\end{array}$ & p value \\
\hline Death & $2(1.6 \%)$ & $2(1.6 \%)$ & 1.0 \\
\hline Atrial fibrillation (postoperative) & $69(54.8 \%)$ & $80(64.0 \%)$ & 0.16 \\
\hline Transient ischemic attack & $3(2.4 \%)$ & $4(3.2 \%)$ & 0.72 \\
\hline Systemic thromboembolism & $1(0.7 \%)$ & $1(0.8 \%)$ & 1.0 \\
\hline Reversible hemiparesis & $1(0.7 \%)$ & $2(1.6 \%)$ & 0.62 \\
\hline Reoperation for degenerated bioprosthesis & $9(7.1 \%)$ & $19(15.2 \%)$ & 0.05 \\
\hline Bleeding requiring hospitalization & $2(1.6 \%)$ & $2(1.6 \%)$ & 1.0 \\
\hline $\begin{array}{l}\text { Functional Class (New York Heart } \\
\text { Association Class II) }\end{array}$ & $9(7.1 \%)$ & $12(9.6 \%)$ & $\mathbf{0 . 0 4}$ \\
\hline
\end{tabular}

Table 2B: Late outcomes (beyond 30 days)

Two patients were lost to follow-up. Follow up was complete in 245 $(99.1 \%)$ patients and yielded 2756.25 patient-years data. Two hundred twenty-four $(91.4 \%)$ patients were in NYHA class I, while $21(8.6 \%)$ were in NYHA class II. The actuarial survival at a median follow-up of 134 (IQR: 99.5-178.5) months was $96.36 \% \pm 0.01 \%$ (95\% CI: 93.1198.10). There was no difference in actuarial survival between the two groups (log rank, p=0.70, Figures 5A, 5B).

However, actuarial survival was significantly lower $(91 \pm 5.7 \%$; $95 \% \mathrm{CI}$ : $82 \%-94 \%)$ in patients reoperated for SVD $(\log$ rank $\mathrm{p}=0.001)$, and in those with an adverse event during late follow-up (90\% $\pm 5.8 \%$; $95 \% \mathrm{CI}$ : 87.1-93.2\%; log rank $\mathrm{p}=0.0008)$, (Figures 6 and 7).

Thirty patients (Group I, $\mathrm{n}=11,8.5 \%$; Group II, $\mathrm{n}=19,14.6 \%$ ) developed severe bioprosthetic degeneration with predominant stenosis between 7 and 10 years after primary tissue valve replacement (Figures 3A, 3B). They underwent redo mitral valve replacement using either Medtronic Mitral Mechanical or St. Jude Mitral Mechanical prostheses as stated above. Intraoperatively, two-dimensional and three-dimensional transesophageal echocardiography demonstrated severe prosthetic valve 
stenosis and no regurgitation. Examination of the explaned bioprostheses revealed severely restricted mobility due to stiffening and calcification of the leaflets. Postoperatively, one patient undergoing redo bioprosthetic replacement required intra-aortic balloon counterpulsation in addition to inotropes for low cardiac output syndrome. Twenty-nine $(96.6 \%)$ patients survived the reoperation, and are presently in New York Heart Association Class I.

There was no difference in the mean diastolic gradient across both bioprosthetic mitral valves in two groups with and without chordal preservation $(2.06 \pm 0.45$ vs $2.09 \pm 0.36 \mathrm{mmHg}$, with and without chordal preservation, $\mathrm{p}=0.08$ ). Between 84 and 100 months of follow-up, valve leaflet thickening with mild prosthetic valve stenosis (Epic: $n=6$ and PERIMOUNT: $\mathrm{n}=2$ ) was seen and being closely followed-up.

Although reoperations for SVD was more among group II (14.6\% vs $8.5 \%$ ), propensity score matching did not exhibit any difference in requirement of reoperations between the groups (SMD: $-0.23, \mathrm{p}=0.5$ ). Patients undergoing reoperations were associated with 3.82 (95\% CI: $1.81,9.56)$ times increased risk of death compared to non-reoperated group, and there was significantly decreased probability of long-term survival (log rank, $\mathrm{p}=0.001)$, (Table 1 ).

The composites of valve-related cumulative events were similar between the two groups (Group I: $16.1 \%, n=21$ vs Group II: 20\%, n=26; SMD $0.18, \mathrm{p}=0.1)$. The actuarial event free survival at a median follow-up of 134 months was $93.06 \pm 0.04 \%$ (95\% CI: 86.1-97.2\%).

Hazard regression for mortality included $\mathrm{HR}(95 \% \mathrm{CI})$ preoperative $\mathrm{CHF}$ 4.70 (1.76-12.56), $\mathrm{p}=0.002$; renal failure 66.91 (12.88-347.59), $\mathrm{p}<0.001$; low LVEF $<0.25,3.76(1.72-7.27), \mathrm{p}=0.004$; and valve-related reoperations $3.82(1.81-9.56), \mathrm{p}=0.001$ (Table 3).

\begin{tabular}{|c|c|c|}
\hline Variables (covariates adjusted) & $\begin{array}{c}\text { Hazard ratio (95\% confidence } \\
\text { interval) }\end{array}$ & p value \\
\hline Renal failure requiring peritoneal/hemodialysis* & $66.91(12.88,347.59)$ & $<0.001$ \\
\hline Redo mitral valve replacement (degenerated prosthesis)* & $3.82(1.81,9.56)$ & 0.001 \\
\hline Left ventricular ejection fraction $<0.25^{*}$ & $3.76(1.72,7.27)$ & 0.004 \\
\hline Congestive cardiac failure (on inotropes, ventilation)* & $4.70(1.76,12.56)$ & 0.002 \\
\hline
\end{tabular}

*Variables with increased risk

Table 3: Risk of 0- to 19-year mortality after bioprosthetic mitral valve replacement by Hazard regression analysis

\section{Comment}

The present study is the first, to our knowledge, to compare Epic with PERIMOUNT mitral bioprosthesis in young rheumatics. This study provides several interesting findings:

i. Despite chordal preservation, on an individualized basis both the bioprostheses had favourable mean pressure gradients and mitral valve areas at short- and long-term follow-up.

ii. No significant difference existed between the two groups of patients with or without chordal preservation, regarding mean pressure gradients (for all valve sizes) on follow-up.

iii. Both Epic and PERIMOUNT bioprostheses exhibited similar valve-related reoperation rates on long-term follow-up (SMD $0.23, \mathrm{p}=0.5)$.

iv. Propensity score matching and multivariable modeling minimizing the biases did not identify mitral bioprosthetic implantation or reoperation as risk factors for mortality in young rheumatics. There was no difference in actuarial survival between the Epic and PERIMOUNT bioprostheses.

According to American College of Cardiology/American Heart Association 2006 guidelines:

\section{Class I:}

A bioprosthesis is indicated for MVR in patients who cannot take warfarin (Level of Evidence: C).[16]

\section{Class IIa:}

1. A mechanical mitral prosthesis is reasonable in patients aged $<65$ years with long-standing atrial fibrillation. (Level of Evidence: C)

2. A mitral bioprosthesis is reasonable in patients $>65$ years. (Level of Evidence: C)
3. A mitral bioprosthesis is reasonable in patients aged $<65$ years in sinus rhythm who elect to receive this valve for lifestyle considerations. (Level of Evidence: C)

Published data indicate that strong consideration should be given to choosing a tissue over a mechanical prosthesis in patients $>60$ years, but the issue remains largely unsettled in patients aged <60-years. [6-9,17] The rationale for these studies is based on improved durability of bioprosthesis, anticipated low risk of reoperation and avoidance of longterm anticoagulation. Data on long-term survival of patients with bioprosthesis, however are conflicting. [5,9,16-21]

The quest to establish a bioprosthesis that is durable for 20- to 25-years continues. [10,17,18-20] Of these, Carpentier-Edwards PERIMOUNT, a second generation bioprosthesis was designed to eliminate SVD which plagued the first-generation prosthesis, while retaining hemodynamic superiority conferred by pericardial valve substitutes.[19]

The St. Jude Medical porcine bioprostheses include Biocor and Epic. Biocor is formulated with no calcium mitigation therapy whereas Epic is treated with Linx AC, an ethanol-based therapy for calcium mitigation. Both valves are tricomposite bioprostheses with low-pressure glutaraldehyde preservation. They have low-profile flexible stent with a pericardial shield designed to aid in valve durability providing a tissueto-tissue contact when the valve opens and closes. $[11,18,20]$

Commercial heart valve companies have sought to promote the utilization of third-generation bioprosthetic valves by promising diminished residual gradients and improved tissue durability.[11,18,20] Several factors complicate the comparison of early hemodynamic performance among devices, including heterogeneity of commercial valve sizers, differing prosthetic leaflet tissue types (bovine vs porcine), varied leaflet preservation/ anticalcification treatment, and the disparate study designs within which devices are compared.

Despite the divergence of data, at a median follow-up of 134 months (IQR 99.5-178.5), the reoperation rate in our study was 8.5\% in Epic and 
$14.6 \%$ in PERIMOUNT arm, while reoperations for SVD was associated with 3.82 times $(95 \%$ CI: 1.81-9.56, $\mathrm{p}=0.001)$ increased risk of death.

All biologic valves are at risk of SVD. The younger the patients are, the earlier the valve degenerates. [11,18,19] Freedom for SVD-related reoperation rates at 10 and 15 years in patients aged $<60$ years has been reported between $71-84 \%$ and $62.6 \%-87.4 \%$ respectively. [11,18,19]

Our actuarial survival at a median follow-up of 134 months was $96.36 \pm 0.01 \%$ which is in accordance with the published investigations of 10 -year survival of $52.3 \%$ with PERIMOUNT and $42 \%-58.8 \%$ with Epic.[8,9,12,18-20] There was no difference in actuarial survival between Epic or PERIMOUNT prosthesis (Log rank $=0.70)$, (Figures $5 \mathrm{~A}, 5 \mathrm{~B})$.

A number of recent articles supported the use of bioprostheses in patients aged <60 years with the argument that bioprostheses reduced the postoperative valve-related complications including SVD and mortality. Myken and associates studied Biocor MVR in 1712 patients with a mean follow-up of 6.2 years. [18] The 20-year freedom from actuarial valverelated mortality was $92.8 \%$ and freedom from SVD was $79.3 \%$. They concluded that bleeding was more hazardous than reoperation.[18] The Biocor MVR durability reported by Pomerant Zeff and associates in 2006 on 546 patients (mean age 48 years) at 15 years was $51.8 \%$ for those aged $<50$ years, $88.7 \%$ for those $51-60$ years, and $84 \%$ for those 61-81 years for reoperative SVD. [20]

An important argument in favour of bioprosthetic valve is the freedom from chronic anticoagulation. It is pertinent to point out that in our study, a significant number of patients with atrial fibrillation with or without LA/LAA clot, history of thromboembolism, and those undergoing surgical LA reduction for giant LA were on low-intensity anticoagulation with an INR between 1.5-2.0. With this strategy, we observed the linearized occurrence rates of composites of valve-related cumulative events (bleeding and thromboembolism) of 1.4 events per 100 patient-years, which is comparable to other reports. [10,15,19,21]

Literature is divided on the issue of ligation of LAA and management of giant LA during mitral valve surgery.[21,22] Studies have shown that LAA plays an important role in genesis of LA thrombus in patients with atrial fibrillation and ligation of LAA during MVR in high-risk population reduces the risk of late thromboembolism and is a recommended procedure in ACC guidelines.[16] However LAA ligation may not provide an adequate protection from thromboembolic events in the absence of effective anticoagulation with warfarin.

In our study, $68.4 \%(n=178)$ were in atrial fibrillation, $36.5 \%(n=95)$ had LA size $>65 \mathrm{~mm}, 22.3 \%(\mathrm{n}=58)$ had LAA/LA clot, 3.1\% $(\mathrm{n}=20)$ had preoperative history of thromboembolism, and $33.1 \%(\mathrm{n}=86)$ had LVEF $<0.25$. No surgery was performed for atrial fibrillation because ventricular rate was well controlled on pharmacological therapy and there was no intolerance of arrhythmia. Eight patients undergoing redo MVR for SVD had large LA clot, atrial fibrillation, low intensity anticoagulation, and unligated LAA. LAA ligation was done in $90.4 \%$ $(n=235)$ patients. Fifty-three patients had amputated LAA during previous surgery and in 25 patients LAA was not ligated due to small size.

Currently, there is no consensus on management of giant LA during mitral valve surgery. Studies have reported surgical mortality between $8 \%$ to $23 \%$ in patients undergoing surgery for mitral valve and giant LA which is unacceptable. [23,24] In our study, 36.5\% ( $\mathrm{n}=95)$ patients underwent LA reduction for giant LA by plicating the inferior and superior LA wall and ligating LAA. We avoided partial excision on superior wall because it carries greater risk for bleeding and atrioventricular node blockade.
Our analysis revealed that over long-term follow-up, valve-related survival, reoperations, and freedom from SVD were marginally superior with Epic group as compared to PERIMOUNT. Although, slight numeric differences were noted in the above parameters favouring Epic, these differences were unlikely to affect clinically significant variables and were statistically insignificant. It is noteworthy that hazard regression analysis did not identify either group or reoperation as risk factors for late mortality. This study can serve as the basis for long-term durability and performance evaluation of both Epic and PERIMOUNT MVR in young rheumatics. $[23,24]$

\section{Study Limitations}

Although the implications of the present findings are multiple, it is important to caution that: i) like other observational cohorts, the results of these analyses may not be generalizable to all young patients who have undergone MVR at other centers, ii) they only apply to valve selection at initial implant operation, iii) may apply only if perioperative mortality of reoperation is low and consistent with modern standards, and iv) should not be extrapolated to patients undergoing double valve replacement. Although our study is limited by its retrospective nature, propensity score analysis provides a balance of two compared groups and attempt to control for the most of the bias in assignment of valve type.

\section{Conclusions}

This study provides some of the robust-evidence available on long-term outcomes after bioprosthetic MVR in rheumatics. Our results indicate excellent durability of both PERIMOUNT and Epic bioprostheses between 8 and 19 years. Although, uncommon, reporting relatively early degeneration in younger rheumatics is warranted to recognize the mechanisms underlying early degeneration of bioprosthetic heart valves. We conclude that present generation mitral bioprostheses are acceptable in young patients providing excellent survival and low composites of valve-related complications. PERIMOUNT prosthesis may not be preferred in patients with small left ventricle.

\section{References}

1. John S, Ravikumar E, Jairaj PS, Chowdhury UK, Krishnaswami $\mathrm{S}$. Valve replacement in the young patient with rheumatic heart disease. Review of a twenty-year experience. J Thorac Cardiovasc Surg 1990;99(4):631-638.

2. Cannegieter SC, Rosendaal FR, Briet E. Thromboembolic and bleeding complications in patients with mechanical heart valve prostheses. Circulation 1994; 89: 635-641.

3. Chowdhury UK, Sharma P, Kumari LS, et al. Thromboembolic and bleeding complications following St. Jude Medical valve replacement. Annals of Short Reports 2018; 1: 1009.

4. Ikonomidis JS, Kratz JM, Crumbley AJ, et al. Twenty-year experience with the St. Jude Medical mechanical valve prosthesis. J Thorac Cardiovasc Surg 2003; 6: 2022-2031.

5. Chowdhury UK, Kumar AS, Airan B, et al. Mitral valve replacement with and without chordal preservation in a rheumatic population: serial echocardiographic assessment of left ventricular size and function. Ann Thorac Surg 2005;79:1926-1933.

6. Ruel M, Kulik A, Lam BK, et al. Long-term outcomes of valve replacement with modern prostheses in young adults. Eur J Cardiothorac Surg 2005;27(3):425-433.

7. Nishimura RA, Otto CM, Bonow RO, et al. AHA/ACC guidelines for the management of patients with valvular heart disease: executive summary: a report of the ACC/AHA Task Force on Practice Guidelines. J Am Coll Cardiol. 2014;63 (22):2438-2488. 
8. Nakano K, Hirahara N, Motomura N, et al. Current status of cardiovascular surgery in Japan, 2013 and 2014: A report based on the Japan Cardiovascular Surgery Database. 4. Valvular heart surgery. Gen Thorac Cardiovasc Surg 2017; 66: 13-18.

9. Stassano P, Tommaso LD, Monaco M, et al. A prospective randomized evaluation of mechanical versus biological valves in patients aged 55 to 70 years. J Am Coll Cardiol 2009;54:18621868.

10. Chowdhury UK, Rizvi A, Narang R, et al. Mitral valve replacement using Carpentier-Edwards pericardial bioprosthesis in patients with rheumatic heart disease aged below 40 years: $17-$ year results. Heart Lung and Circulation 2018; 27: 864-871.

11. Jamieson WRE, Lewis CTP, Sakwa MP, et al. St Jude Medical Epic porcine bioprosthesis: results of the regulatory evaluation. $\mathrm{J}$ Thorac Cardiovasc Surg. 2011;141:1449-54.e2.

12. Grunkemeier GL, Jamieson WR, Miller DC, Starr A. Actuarial versus actual risk of porcine structural valve deterioration. J Thorac Cardiovasc Surg. 1994;108:709-718.

13. Chowdhury UK, Venkataiya JKH, Patel CD, et al. Serial radionuclide angiographic assessment of left ventricular ejection fraction and regional wall motion after mitral valve replacement in patients with rheumatic disease. Am Heart J, 2006; 152: 12011207.

14. Edmunds LH Jr, Clark RE, Cohn LH, et al. Guidelines for reporting morbidity and mortality after cardiac valvular operations.J Thorac Cardiovasc Surg. 1988; 96: 351-353

15. Cheitlin MD, Armstrong WF, Aurigemma GP, et al. ACC/AHA/ASE 2003 guideline update for the clinical application of echocardiography: summary article: a report of the ACC/AHA/ASE Committee to Update the 1997 Guidelines for the Clinical Application of Echocardiography). J Am Coll Cardiol 2003; 42: 954-970.

16. Bonow RO, Carabello BA, Chatterjee K, et al. ACC/AHA 2006 guidelines for the management of patients with valvular heart disease: a report of the ACC/AHA Task Force on Practice Guidelines. J Am Coll Cardiol. 2006;48:e1-148.

17. Vahanian A, Alfieri O, Andreotti F, et al; Joint Task Force on the Management of Valvular Heart Disease of the ESC, EACTS. Guidelines on the management of valvular heart disease. Eur Heart J. 2012;33(19):2451-2596.

18. Myken PS, Bech-Hansen O. A 20-year experience with the Biocor porcine bioprosthesis. J Thorac Cardiovasc Surg 2009; 137:76-81.

19. Marchand MA, Aupart MR, Norton R, et al. Fifteen-year experience with the mitral Carpentier-Edwards PERIMOUNT pericardial bioprosthesis. Ann Thorac Surg. 2001;71(5 Suppl.): S236-9.

20. Pomerantzeff PMA, Brandao CMA, Albuquerque JMCA, et al. Long-term follow up of the Biocor porcine bioprosthesis in the mitral position. J Heart Valve Dis. 2006;15:763-67.

21. Sankhyan LK, Chowdhury UK, Rizvi A, et al. Evaluation of thromcoembolism and valve thrombosis in patients with rheumatic heart disease undergoing mitral tissue valve replacement in the presence of atrial fibrillation with or without left atrial clot: Review of a 17-years' experience. World J Surg Surgical Res 2018; 1: 1042.

22. Juratli N, Wilkoff B, Tchou $\mathrm{P}$, et al. Left atrial appendage ligation during mitral valve surgery may increase the risk of late thromboembolic event. J Am Coll Cardiol. 2002;39:85A.

23. Kawazoe K, Beppu S, Takahara Y, et al. Surgical treatment of giant left atrium combined with mitral valvular disease. Plication procedure for reduction of ompression to the left ventricle, bronchus and pulmonary parenchyma. J Thorac Cardiovasc Surg 1983; 85: 885-892.

24. Piccoli GP, Massini C, Di Eusanio G, et al. Giant left atrium and mitral valve disease: early and late results of surgical treatment in 40 cases. J Cardiovasc Surg (Torino) 1984; 25: 328-336.
This work is licensed under Creative Commons Attribution 4.0 License
To Submit Your Article Click Here: Submit Manuscript

DOI:10.31579/2641-0419/142
Ready to submit your research? Choose Auctores and benefit from:

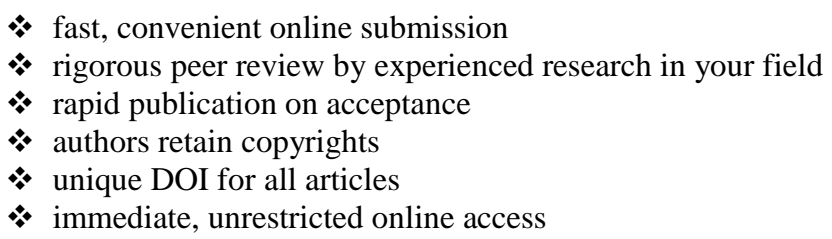

At Auctores, research is always in progress.

Learn more www.auctoresonline.org/journals/clinical-cardiology-andcardiovascular-interventions 